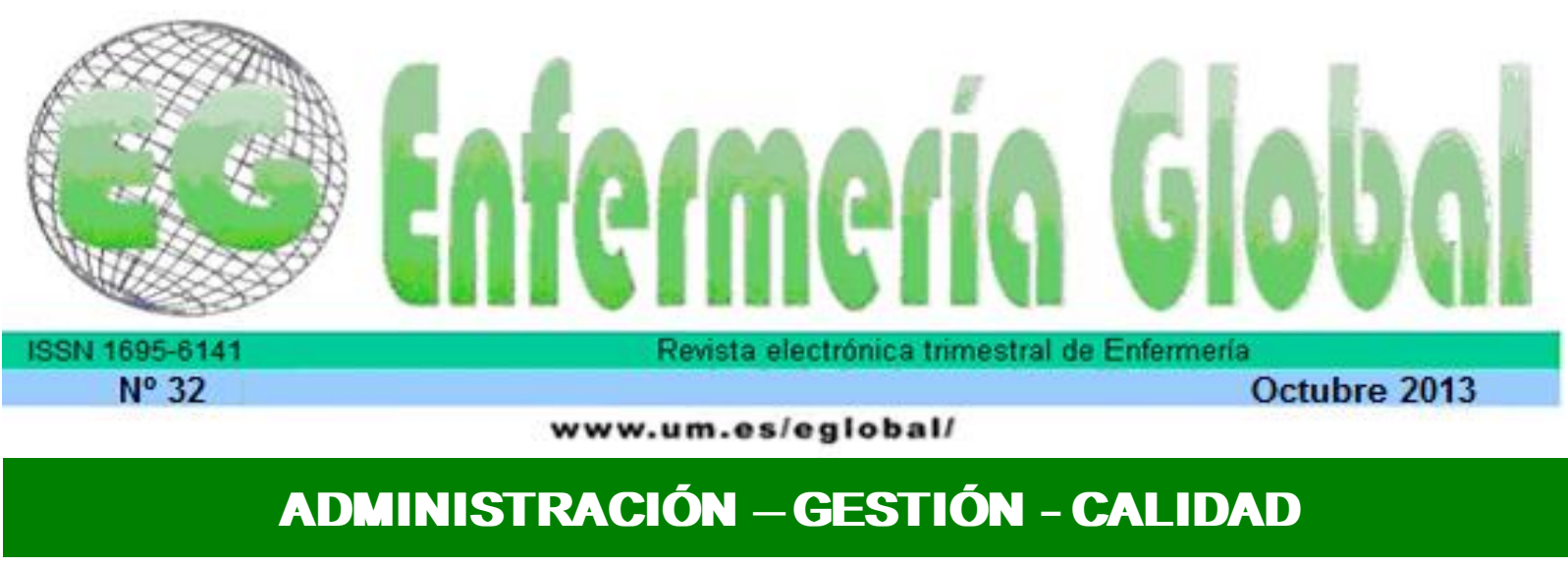

\title{
Relación entre los problemas de salud de los mayores dependientes y la formación de los cuidadores informales
}

Relation between health problems of elderly dependent and training of informal caregivers

\author{
*Guerra Martín, María Dolores **Zambrano Domínguez, Encarnación María \\ * Departamento de Enfermería, Facultad de Enfermería, Fisioterapia y Podología, Universidad de \\ Sevilla. E-mail: guema@us.es
}

Palabras clave: Cuidadores; anciano; dependencia; capacitación.

Keywords: Caregivers; aged; dependency; training.

\section{RESUMEN}

Objetivo: Estudiar la relación entre los problemas de salud de los mayores dependientes y la formación de los cuidadores informales.

\begin{abstract}
Material y métodos: Se presenta un estudio exploratorio, descriptivo, de corte transversal, seleccionando a 41 cuidadores mediante muestreo no probabilístico intencional. Se ha diseñado un cuestionario basado en la bibliografía existente y cuya validez de contenido ha sido consensuada por expertos. Para el análisis de datos se ha utilizado SPSS versión 18.0.
\end{abstract}

Resultados: Un 41,5\% de los cuidadores informales recibieron algún tipo de formación; un 92,7\% de cuidadores plantean que necesitan formación en relación con los problemas de salud de las personas cuidadas y un $58,5 \%$ de mayores han sido cuidadas por cuidadores que no han recibido formación.

Conclusiones: Es significativo, que en nuestro estudio hay más cuidadores sin formación cuidando personas con problemas de salud, que con formación específica; siendo necesario que los cuidadores informales principales puedan asistir a los programas formativos sobre los problemas de salud y sobre los cuidados que requieran las personas mayores que cuidan, por lo que se plantea como estrategia de mejora, que desde la gestión sanitaria se proporcionen los recursos y medios que necesiten los cuidadores, y se potencie el uso de herramientas formativas (foros, chats, correos electrónicos, tuenti, facebook, blog, twitter, Skype, entre otros), que pueden plantearse como alternativa a las sesiones presenciales.

\section{ABSTRACT}

Aim: To study the relationship between the health problems of elderly dependent and training of informal caregivers. 
Methods: We present an exploratory, descriptive cross-sectional, selecting 41 caregivers through intentional non-probability sampling. He has designed a questionnaire based on the literature and content validity has been agreed upon by experts. For data analysis has been used SPSS version 18.0.

Results: $41.5 \%$ of informal caregivers received some training, $92.7 \%$ of caregivers state that need training regarding the health problems of people cared and $58.5 \%$ of seniors have been maintained for caregivers who are not trained.

Conclusions: It is significant that in our study there are more untrained caregivers caring for people with health problems than with specific training, being necessary to the main informal cares to attend training programs on issues of health care and requiring older people who care for what is proposed as a strategy for improvement, health management since they provide the resources and facilities needed by caregivers, and enhance the use of training tools (forums, chats, emails, Tuenti, facebook, blog, twitter, Skype, etc.), which can arise as an alternative to face sessions.

\section{INTRODUCCIÓN}

El aumento de la población mayor está generando un aumento de las tasas de dependencia, siendo un problema que está adquiriendo gran relevancia en la sociedad actual, y por ello, es de especial preocupación la necesidad creciente de asistencia y cuidados de larga duración por parte de personas mayores que se encuentran en situación de dependencia ${ }^{(1)}$.

Este acrecentamiento de la población mayor en situación de dependencia, condiciona la necesidad cada vez mayor, de un cuidador que satisfaga las necesidades y que brinde la atención que estas personas no pueden proveerse por sí mismas; la principal fuente proveedora de cuidados suele ser la familia y dentro de la misma, es solo una persona la que se hace cargo de proveer los cuidados requeridos, denominándose cuidador informal principal ${ }^{(2)}$.

En España las relaciones familiares juegan un papel muy importante, pues son los miembros de las familias los que asumen el rol de cuidador cuando en ella existe una persona mayor en situación de dependencia que requiere cuidados, es por tanto, la red familiar la que se encuentra al frente de la atención requerida ${ }^{(3)}$, de ahí la importancia de divulgar los distintos programas de formación e información para poder proveer mejores cuidados a las personas dependientes ${ }^{(4)}$.

En distintos estudios los cuidadores informales principales manifiestan la necesidad de adquirir conocimientos y formación en relación a los cuidados básicos generales que requiere la persona cuidada y técnicas para mejorar el cuidado de ellos mismos $^{(5,6)}$.

A pesar de existir intervenciones dirigidas a atender a los cuidadores en las diferentes comunidades autónomas en España, y especialmente en Andalucía ${ }^{(7,8)}$, que ponen énfasis en la elaboración de acciones formativas, en diferentes investigaciones recientes se recoge la escasa formación que generalmente reciben los cuidadores informales principales ${ }^{(5,6,9)}$, que aseguran así una provisión de cuidados de calidad y que afecta tanto a la salud de la persona cuidada como a la de ellos mismos ${ }^{(10)}$.

En diferentes investigaciones, se refleja que gran parte de los cuidadores informales principales no reciben ayuda informal por parte de otro familiar ni de otro servicio ${ }^{(2,11)}$, siendo necesario que tanto el cuidado principal como la familia, cuenten con el apoyo, ayuda, preparación y formación para proporcionar el soporte y apoyo afectivo, y 
material necesarios para atender a las necesidades de las personas en situación de dependencia $^{(12)}$.

Se considera que el desarrollo de programas formativos es escaso, y que en general satisfacen distintas actividades de la persona cuidada con insuficiente competencia técnica, es por lo que los cuidadores informales demandan actividades formativas relacionadas tanto con técnicas específicas (curas, administración medicación, alimentación) como técnicas de promoción y prevención de la salud (cambios posturales y ejercicios de movilidad); surgiendo así la necesidad de dar apoyo emocional y formación a las cuidadoras informales ${ }^{(5)}$.

Hay que proporcionar una formación adecuada a las familias proveedoras de cuidados informales, ya que estas quieren saber cómo afrontar las consecuencias personales que se derivan del cuidado; la atención al cuidador debería extenderse a lo largo de todo el proceso de cuidado, ofreciendo un asesoramiento continuado y personalizado, que no se trataría por tanto, de ofrecer un simple curso de formación ${ }^{(13,14)}$.

Es importante tener en cuenta que el cuidador familiar no tiene por qué tener formación en salud por lo que los cuidados están constituidos por sus experiencias de la vida diaria, lo que conlleva una fuente de afecto y reciprocidad a largo plazo y la apertura a todos los conocimientos de las personas y de los contextos ${ }^{(9)}$.

En los cuidados familiares a pacientes con Alzheimer, la mayoría de los cuidadores no tienen una formación previa sobre los cuidados específicos, teniendo las personas cuidadoras que aprenden a cuidar por sí mismas, de sus experiencias como cuidadores y de la práctica del día a día y a través de aciertos y errores ${ }^{(15)}$.

Para la revisión de la literatura se han manejado distintas bases de datos, las estrategias de búsqueda en las bases de datos nacionales (IME, ISOC y CUIDEN) han sido: enfermer ${ }^{*} Y$ (forma* $O$ educa* $O$ aprend ${ }^{*}$ ) $Y$ cuidad $^{*} Y$ (adult* $^{*}$ O mayor $\mathrm{O}$ ancian* $^{*} Y$ depend $^{*}$, desde el año 2005. En las bases de datos internacionales (MEDLINE, SCOPUS y CINHAL): (caregiv* OR caretak $^{*}$ ) AND (educat* OR form* OR learn*) AND dependen*, desde el año 2005 y por la edad a mayores de 65 años.

En este trabajo se plantea estudiar la relación entre los problemas de salud de los mayores dependientes y la formación de los cuidadores informales, siendo el presente trabajo fruto de una investigación más amplia, de la cual se ha generado otro trabajo ${ }^{(16)}$ con el abordaje de otros aspectos no incluidos en el presente estudio.

\section{MATERIAL Y MÉTODOS}

Se trata de un estudio exploratorio, descriptivo, de corte transversal, realizado durante seis meses (2010/2011), en la Unidad de Gestión Clínica (UGC) Nuestra Señora de las Nieves de la Zona Básica de Salud de Los Palacios y Villafranca de Sevilla (España), teniendo esta población un 10,5\% de personas mayores de 65 años o más edad. En esta UGC la población asciende a 597 cuidadores, de ellos, 488 cuidan a una persona mayor de 65 años en situación de dependencia.

La muestra elegida mediante muestreo no probabilístico intencional, ha estado formada por 41 cuidadores informales principales incluidos en el registro del Programa de Cuidadores de Atención Primaria de la UGC. Se establecieron como 
criterios de inclusión a los cuidadores informales principales de personas mayores de 65 años en situación de dependencia que llevaran al menos dos meses como cuidadores, en caso que existiera más de un cuidador informal, se elegiría aquel cuidador que llevase más tiempo dedicándose al cuidado diario de la persona cuidada, y por último, se exigía la aceptación de los cuidadores a participar en el estudio. La inexistencia de un cuidador informal principal se estableció como criterio de exclusión.

Las variables analizadas relacionadas con el cuidador informal principal fueron las siguientes: edad, género, estado civil, situación laboral, parentesco/relación con la persona cuidada, apoyo recibido por parte de una persona u otro recurso que le ayude a cuidar, ayuda de la que dispone para cuidar, necesidad de recibir formación o seguir formándose, causa por la que considera importante o no formarse, posibilidad de recibir formación, causa por la que tiene o no posibilidad de formarse, formación recibida y formación recibida en relación con los cuidados que precisa la persona cuidada. Las variables del estudio que estaban relacionadas con la persona mayor cuidada eran la edad, género, estado civil y problema de salud por el cual tiene la dependencia.

Para la recogida de datos se diseñó un cuestionario basado en la literatura científica $^{(3,17,18)}$ y para la validez de contenido se realizó un consenso de expertos ${ }^{(19-}$ 23). El cuestionario administrado mediante entrevista personal a los cuidadores, constaba de dos apartados, uno referido al cuidador que contenía diecisiete preguntas, de las cuales se han utilizado para este estudio nueve preguntas; y otro, referido a la persona mayor dependiente, de cinco preguntas, cuatro de ellas de formulación cerrada y una abierta donde la Enfermera Gestora de Casos detallaba los problemas de salud.

Se ha utilizado para el análisis de los datos el paquete estadístico Statistical Package for the Social Sciences (SPSS) versión 18.0. Para las variables de tipo cualitativo se elaboraron tablas de distribución de frecuencias y porcentajes, y para las variables cuantitativas se calcularon las medidas de centralización y dispersión. Se calculó la media y la desviación típica en aquellas distribuciones que se muestran simétricas, y en aquellas distribuciones que se mostraron asimétricas se determinó la mediana y el recorrido intercuartílico $\left(\mathrm{P}_{25}, \mathrm{P}_{75}\right)$. En todas ellas se calcularon los intervalos de confianza al $95 \%$. Para valorar la relación entre dos variables de tipo cualitativo se realizaron tablas de contingencia y se aplicó el test de Chi-cuadrado o la probabilidad exacta de Fischer (tablas $2^{\star} 2$ grupos pequeños). Para valorar la relación entre dos variables de tipo cuantitativo se calculó el coeficiente de correlación de Pearson o Rho-Spearman según criterio de aplicación. Para analizar la relación entre una variable cualitativa dicotómica y una cuantitativa se realizó el test de la t de Student una vez validado los requisitos de aleatoriedad, normalidad e igualdad de varianza. En el caso de no cumplirse el requisito de igualdad de varianza, se realizó la t de Student con la corrección de Welch, y en caso de no cumplirse el requisito de normalidad, se realizó la prueba no paramétrica U- Mann Withney.

Este estudio obtuvo el informe positivo de las Comisiones de Ética de la Universidad de Sevilla y del Comité de Ética e Investigación Sanitaria del Distrito de Atención Primaria de Salud Sevilla Sur (España), garantizando la confidencialidad y el anonimato, habiendo solicitando el consentimiento informado por escrito de todos los cuidadores participantes. 


\section{RESULTADOS}

La edad media de los cuidadores es de 48,8 $(7,8)$ IC95\% $(46,4-51,3)$ años; un $87,8 \%$ de los cuidadores están casados; el 12,2\% de los cuidadores son hombres; un $80,5 \%$ se encuentran desempleados; un $68,3 \%$ son las hijas de la persona cuidada. En la figura 1 , se presentan los resultados del tipo de ayuda de la que disponen los cuidadores informales que contestaron que disponían de alguna persona u otro recurso que le ayuda a cuidar.

Figura 1. Tipo de ayuda de los cuidadores informales.

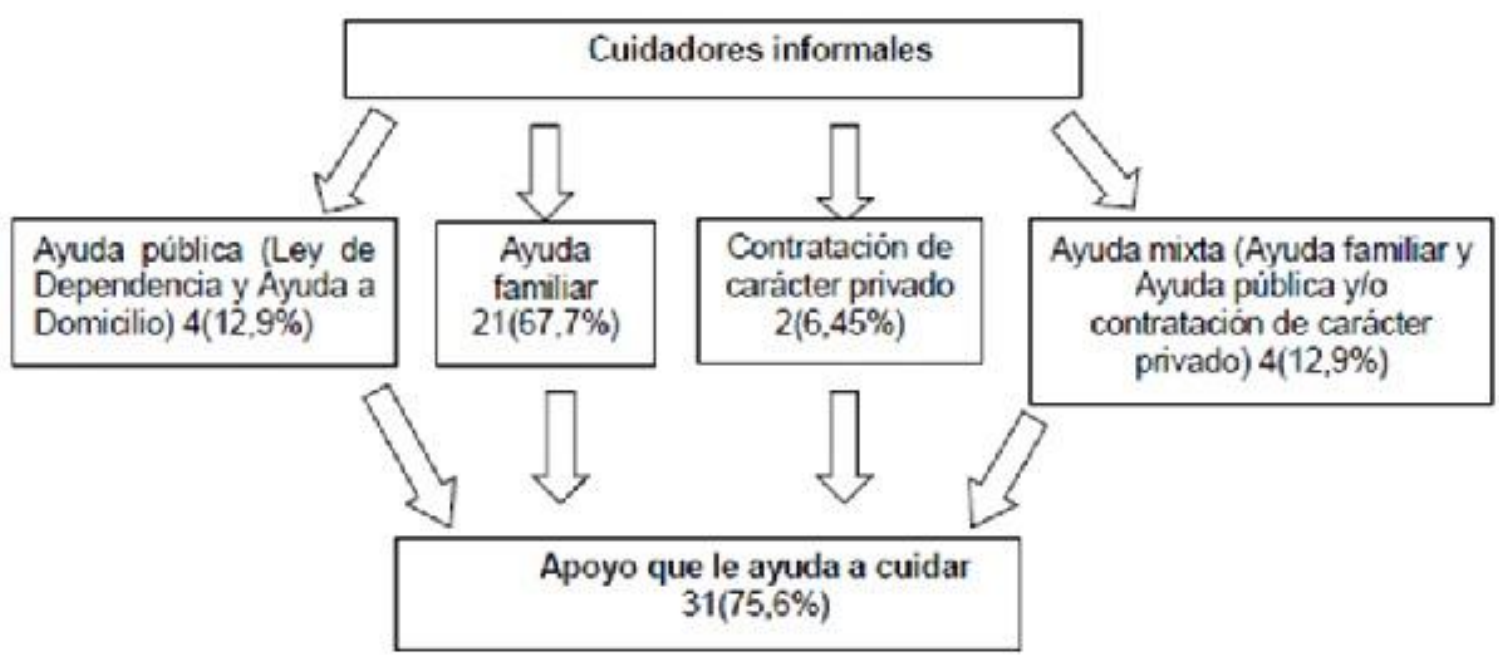

Fuente: Elaboración propia.

Un $41,5 \%$ de las personas cuidadoras respondieron que sí habían recibido algún tipo de acción formativa. En la pregunta que hace referencia a la importancia de formarse (o seguir formándose) sobre el/los problemas y/o cuidados que necesita la persona cuidada, de las 41 personas cuidadoras $38(92,7 \%)$ manifestaron que sí es necesaria la formación. Entre las causas por la que los cuidadores consideran que es importante o no recibir formación se destaca que $24(58,5 \%)$ de los entrevistados creen que es necesario formarse por la necesidad que tienen de aumentar los conocimientos previos, $8(19,5 \%)$ consideran que es importante recibir formación para mejorar la calidad de los cuidados prestados, $6(14,6 \%)$ de las personas cuidadoras tienen la necesidad de recibir formación por falta de conocimientos previos, y el resto de los cuidadores manifiestan que no es necesario recibir formación porque opinan que tienen suficientes conocimientos previos para prestar los cuidados.

De los 41 cuidadores, $26(63,4 \%)$ de ellos han respondido que sí tendrían posibilidad de recibir formación. De éstos, $15(36,6 \%)$ cuidadores manifiestan que disponen de una persona que le supla mientras se forman, el 26,9\% disponen de tiempo para formarse y 2 (4,9\%) personas disponen de un tiempo limitado para ello. El $36,5 \%$ de los cuidadores han respondido que no tendrían posibilidad de recibir formación, de éstos $14(34,1 \%)$ manifiestan que no disponen de tiempo para ello y $1(2,4 \%)$ cuidador no dispone de una persona que le supla mientras se forma.

La edad media de las personas cuidadas es de $82,1(9,5)$ IC95\% $(79,1-85,1)$ años. La persona cuidada más joven tiene una edad de 65 años, y 96 años la persona más anciana. Un $75,6 \%$ de los cuidadores estudiados son mujeres. Un $58,5 \%$ de las personas mayores dependientes cuidadas son viudas, 15 (36,6\%) están casadas, y el resto son solteras. En cuanto al problema de salud que presentan las personas 
mayores cuidadas, y que ha sido la causa de la situación de dependencia en el que se encuentran, un 26,8\% padece la Enfermedad de Alzheimer, seguida del accidente cerebrovascular $10(24,4 \%)$ (AVC), el resto de los problemas de salud se deben a otras causas (Tabla I).

Tabla I. Problemas de salud que se presentan en la persona mayor dependiente.

\begin{tabular}{|l|c|c|}
\hline PROBLEMAS DE SALUD & $\mathbf{n}$ & $\%$ \\
\hline Enfermedad de Alzheimer & 11 & 26,8 \\
\hline AVC & 10 & 24,4 \\
\hline Proceso de envejecimiento & 5 & 12,2 \\
\hline Demencia senil & 4 & 9,8 \\
\hline Problemas degenerativos articulares & 4 & 9,8 \\
\hline Inmovilización & 3 & 7,3 \\
\hline Enfermedad de Parkinson & 2 & 4,9 \\
\hline Ceguera & 1 & 2,4 \\
\hline Cáncer & 1 & 2,4 \\
\hline
\end{tabular}

Fuente: Elaboración propia.

A continuación, se exponen los resultados que se obtienen tras hacer un análisis bivariado entre la variable formación recibida por los cuidadores informales con otras variables del estudio:

Un $82,4 \%$ IC95\% $(56,6-92,2)$ de las personas cuidadoras que han recibido formación disponen de algún tipo de ayuda. Sin embargo, 17 (70,8\%) IC95\% (48,9-87,4) de los cuidadores que no han recibido formación disponen de algún tipo de ayuda. No se han encontrado diferencias estadísticamente significativas entre la formación recibida y la ayuda de la que disponen los cuidadores informales principales para cuidar, $\mathrm{p}=0,397$.

De los 21(87,5\%) IC95\% (67,6-97,3) cuidadores que no han recibido formación, consideraron que era necesario recibirla. No se encontró relación estadísticamente significativa entre la variable formación recibida y necesidad de recibir formación, $\mathrm{p}=$ 0,130 .

De los cuidadores informales principales que recibieron formación 17(41,5\%), tenían posibilidad de recibirla: 12 (70,6\%) IC95\% (44,1-89,7). Sin embargo, 5 (29,4\%) IC95\% $(10,3-56,0)$ de las personas cuidadoras que recibieron formación no tenían posibilidad de recibirla y $14(58,3 \%)$ IC95\% $(33,0-75,0)$ no recibieron formación, teniendo posibilidades de recibirla. Podemos afirmar que no existe relación entre la variable formación recibida y la variable posibilidad de recibir formación, p=0,422.

De los cuidadores que recibieron formación, 12 (70,6\%) IC95\% (44,1-89,7) lo ha hecho sin disponer de tiempo y/o de persona que le supla mientras se forma. De las personas cuidadoras que no han recibido formación, $10(41,7 \%)$ IC95\% $(19,6-63,5)$ tienen tiempo para ello y/o disponen de una persona que le supla mientras se forman. No existiendo diferencia estadísticamente significativa entre las causas por las que 
tiene o no posibilidad de recibir formación entre los cuidadores informales principales que recibieron formación frente a los que no la recibieron, $p=0,422$.

De los cuidadores informales que disponían de ayuda para cuidar, 21 (67,7\%) IC95\% $(46,7-85,8)$ tenían posibilidad de recibir formación. De los cuidadores que no disponían de ayuda, 5 (50\%) IC95\% (18,7-81,3) tenían posibilidad de recibir formación. No existiendo diferencia estadísticamente significativa entre la variable ayuda recibida y la de posibilidad de recibir formación, $p=0,311$.

Un $41,2 \%$ IC95\% $(18,4-67,1)$ de los cuidadores informales principales que recibieron formación cuidaban a una persona mayor en situación de dependencia con enfermedad de Alzheimer (Tabla II).

Tabla II. Relación de la formación recibida por el cuidador con el problema de salud de la persona cuidada.

\begin{tabular}{|l|c|c|}
\hline PROBLEMAS DE SALUD & FORMACIÓN & NO FORMACIÓN \\
\hline Enfermedad de Alzheimer & $41,2 \%$ & $16,7 \%$ \\
\hline AVC & $17,6 \%$ & $29,2 \%$ \\
\hline Proceso de envejecimiento & $0,0 \%$ & $20,8 \%$ \\
\hline Demencia senil & $12,8 \%$ & $8,3 \%$ \\
\hline Problemas degenerativos articulares & $11,8 \%$ & $8,3 \%$ \\
\hline Inmovilización & $0,0 \%$ & $12,5 \%$ \\
\hline Enfermedad de Parkinson & $5,9 \%$ & $4,2 \%$ \\
\hline Ceguera & $5,9 \%$ & $0,0 \%$ \\
\hline Cáncer & $5,9 \%$ & $0,0 \%$ \\
\hline
\end{tabular}

Fuente: Elaboración propia.

Un $41,5 \%$ (17) personas mayores dependientes han sido cuidadas por cuidadores que han recibido formación, 8 de las personas cuidadas tenían un solo problema de salud, 7 tenían dos y 2 presentaban 3 problemas. Un 58,5\% (24) personas mayores dependientes han sido cuidadas por cuidadores que no han recibido formación, 15 tenían un solo problema de salud, 8 tenían dos y 1 presentaba 3 problemas (Tabla III). 
Tabla III. Necesidad de recibir formación de los cuidadores en relación con los problemas de salud.

\begin{tabular}{|l|c|c|c|}
\hline \multirow{2}{*}{ PROBLEMAS DE SALUD } & \multicolumn{3}{|c|}{ Necesidad de recibir formación } \\
\cline { 2 - 4 } & SI & NO & Total \\
\hline AVC & 9 & 1 & 10 \\
\hline Alzheimer & 11 & 0 & 11 \\
\hline Parkinson & 2 & 0 & 2 \\
\hline Demencia senil & 4 & 0 & 4 \\
\hline Cáncer & 1 & 0 & 1 \\
\hline $\begin{array}{l}\text { Proceso de } \\
\text { envejecimiento }\end{array}$ & 3 & 2 & 5 \\
\hline $\begin{array}{l}\text { Problemas degenerativos } \\
\text { articulares }\end{array}$ & 4 & 0 & 4 \\
\hline Ceguera & 1 & 0 & 1 \\
\hline Inmovilización & 3 & 0 & 3 \\
\hline Total & 38 & 3 & 41 \\
\hline
\end{tabular}

Fuente: Elaboración propia.

\section{DISCUSIÓN}

El perfil de la persona mayor en situación de dependencia que necesita de un cuidador, en un elevado porcentaje son mujeres, viudas y casadas en su mayoría, datos que no difieren de los resultados obtenidos en la encuesta realizada por el IMSERSO $^{(6)}$. La edad media de las personas mayores cuidadas de la muestra que están recibiendo cuidados informales, es de 82,1 años, no discrepando de los datos que aportan otros estudios ${ }^{(3)}$ sobre la edad de las personas mayores dependientes de nuestro país que reciben cuidados informales.

Las tareas de cuidado recaen en un porcentaje elevado en las mujeres $(87,8 \%)$, resultados similares obtienen los autores de otros estudios ${ }^{(2,12,24)}$. La edad media obtenida de los cuidadores del estudio, es de 48,8 años, lo cual difiere de otras investigaciones donde la edad es más elevada(11). La mayoría de informales principales están casados y desempleados, coincidiendo con otros estudios ${ }^{(2,12)}$.

Un número elevado de cuidadores $(75,6 \%)$ recibe algún tipo de apoyo que le facilite en la medida de lo posible los cuidados llevados a cabo, lo que difiere de otro estudio realizado $^{(2)}$. El tipo de ayuda que generalmente reciben las personas cuidadoras suele ser una ayuda familiar, resultado que coincide con el estudio llevado a cabo por el Ministerio de Trabajo y Asuntos Sociales, donde se expone que el tipo de apoyo recibido es también, familiar ${ }^{(3)}$.

Las personas cuidadoras que han recibido algún tipo de formación, cuidan a una persona mayor dependiente con la enfermedad de Alzheimer. Este dato difiere de la bibliografía consultada, ya que se centran en el estudio de la formación de los cuidadores de personas mayores con Alzheimer, donde se recoge que la formación de éstos es escasa ${ }^{(15)}$.

Se ha detectado un elevado porcentaje de cuidadores que manifiestan querer recibir formación o seguir formándose sobre el/los problemas y/o cuidados que precisa la 
persona a la que cuidan $(92,7 \%)$. Esta necesidad sentida por los cuidadores también se observa en otras investigaciones ${ }^{(5,6,13)}$.

Cuando se le consulta a los cuidadores informales principales por el motivo importante para formarse o seguir formándose, la mayoría quiere aumentar sus conocimientos, en un porcentaje menor manifiestan que se mejora la calidad de los cuidados prestados $^{(10)}$ y otros, para cubrir las necesidades de la persona cuidada ${ }^{(5)}$.

Sobre la posibilidad de recibir formación, en el caso de los cuidadores que contestan afirmativamente, el motivo principal es porque tienen una persona que le suple mientras se forman; y de los que contestan que no, mayoritariamente es porque no tienen tiempo. Al contrastar la pregunta sobre si dispone de alguna persona $u$ otro recurso que le ayude a cuidar con la pregunta sobre la posibilidad de recibir formación, no se observa ninguna relación, por lo que creemos conveniente plantear más estudios encaminados a conocer la posible relación entre estas dos variables.

\section{CONCLUSIONES}

La enfermedad de Alzheimer es uno de los problemas de salud que mayoritariamente tienen las personas mayores dependientes, por lo que nos parece interesante reseñar el metaanálisis realizado por Hernández y Cibanal, donde se plantea que los cuidadores tienen falta de formación con respecto a los cuidados que requieren los enfermos de Alzheimer ${ }^{(15)}$.

En nuestro estudio hay más cuidadores sin formación cuidando personas con problemas de salud, que con formación específica; por lo que se plantea la necesidad de que los cuidadores informales principales puedan formarse sobre los problemas de salud y sobre los cuidados que requieran las personas mayores que cuidan. Es por lo que se plantea como estrategia de mejora que desde la gestión sanitaria se proporcionen los recursos y medios que necesiten los cuidadores, y la necesidad de evaluar los programas formativos que se realicen desde las distintas instituciones, ya que pueden contribuir al mejoramiento de los cuidados y de la calidad de vida ${ }^{(25)}$.

Por último, decir que existe una amplia oferta formativa proporcionada por los profesionales sanitarios de Atención Primaria y un bajo nivel de formación de los cuidadores sobre los problemas de salud de las personas mayores dependientes que cuidan, junto con una baja asistencia a las acciones formativas; por lo que es necesario no solo continuar con la oferta de programas formativos y guías prácticas para estos cuidadores; sino también, generar mecanismos eficaces para que los cuidadores puedan asistir a la formación. Como alternativa para eliminar la barrera de la presencialidad de las sesiones, se plantea el fomento del uso de las tecnologías de la información y comunicación (foros, chats, correos electrónicos, tuenti, facebook, blog, twitter, Skype, entre otros).para las actividades formativas de estos cuidadores.

\section{BIBLIOGRAFÍA}

1. Doncel LV y Gutiérrez T. Las personas mayores y su situación de dependencia en España. Mayores y dependencia: Un enfoque sociológico [serial on the Internet] 2006 [cited 2013 Mar 7]; 104-44. Available from: URL:

http://vlex.com/vid/aspectos-demograficos-poblacion-443009 
2. Velasco E. Educación grupal a cuidadores. In: Sánchez MB, Gómez J, Duarte G. editors. Educación para la salud. Madrid: FUDEN; 2008. p. 367-82.

3. Libro Blanco de la Dependencia. Cuidados de larga duración a cargo de la familia: el apoyo Informal. Capítulo 3. Madrid: Ministerio de Trabajo y Asuntos Sociales; 2005. p. 167-26.

4. Red2Red Consultores. Valoración económica de los cuidados a personas dependientes. Zerbitzuan 45. Revista de servicios sociales. 2009; 73(6): 73-81.

5. Gil-García E, Escudero-Carretero M, Prieto-Rodríguez MA, et al. Vivencias, expectativas y demandas de cuidadoras informales de pacientes en procesos de enfermedad de larga duración. Enfermería Clínica. 2007; 15(4): 220-6.

6. IMSERSO. Cuidado a las personas mayores en los hogares españoles. El entorno familiar. Colección Estudios Serie de Dependencia. Madrid: IMSERSO; 2005.

7. Consejería para la Igualdad y Bienestar Social. Cuidabús. El cuidado en el entorno familiar de personas en situación de dependencia [serial on the Internet] 2010 [cited 2013 Mar 7]. Available from: URL: https://www.juntadeandalucia.es/igualdadybienestarsocial/extranet/cuidabus/pres entacion.php

8. Consejería para la Igualdad y Bienestar Social. Talleres de formación presenciales dirigidos a personas cuidadoras. El cuidado en el entorno familiar de personas en situación de dependencia [serial on the Internet] 2010 [cited 2013 Mar 7]. Available from: URL: http://www.juntadeandalucia.es/igualdadybienestarsocial/export/Dependencias/H TML/PLANFORMACION2010/index.html

9. Giraldo $\mathrm{Cl}$, Franco GM, Correa LS, et al. Cuidadores familiares de ancianos: Quiénes son y cómo asumen este rol. Revista de la Facultad Nacional de Salud Pública. 2005; 23(2): 7-15.

10. Burgos $\mathrm{P}$, Figueroa V, Fuentes $\mathrm{M}$, et al. Caracterización y nivel de conocimiento del cuidador informal de usuarios con dependencia severa adscrito al Centro de Salud Violeta Parra-Chillán. Theoria. 2008; 17(2): 7-14.

11. IMSERSO. Encuesta de Apoyo Informal a los mayores en España. Madrid: IMSERSO. 2004.

12. Bódalo-Lozano E. Cambios en los estilos de vida de las cuidadoras de personas dependientes. Portularia. 2010; 10(1): 85-97.

13. Berjano E. Dependencia y Calidad de vida en las personas mayores. Revista Multidisciplinaria de Gerontología. 2005; 15(3):144-54.

14. Casado-Mejías R., Ruiz-Arias E. y Solano-Parés A. El cuidado familiar prestado por mujeres inmigrantes y su repercusión en la calidad del cuidado y en la salud. Gac Sanit. 2012;26(6):547-53.

15. Hernández ME, Cibanal JL. El Aprendizaje de cuidados familiares a pacientes con Alzheimer. Revisión Bibliografía. Cultura de los cuidados 2008. Año XII. 1er Semestre $\mathrm{n}^{\circ} 23$.

16. Zambrano-Domínguez E. M. y Guerra-Martín M. D. Formación del cuidador informal: relación con el tiempo de cuidado a personas dependientes mayores de 65 años. Aquichán. 2012, 12(3): 241-51.

17. Regueiro AA, Gómara SM; Ferreiro MC, et al. El Cuidador Principal de nuestros ancianos dependientes. Salud Rural. 2006; 23 (11): 39-46.

18. Sirpa Salin M.K. y Astedt-Kurki P. Informal carers of older family members: how they manage and what support they receive from respite care. Journal of Clinical Nursing. 2009; 18: 492-01 
19. Fuentesalz Gallego C., Roca Roger M., Úbeda Bonet I., et al. Validation of a questionnaire to evaluate the quality of life of nonprofesional caregivers of dependent persons. Journal of Advaced Nursing. 2001; 33(4): 548-554.

20. Hernández, R., Fernández-Collado, C. y Baptista, P. Metodología de la investigación, 4 ed. México: McGraw-Hill.Carretero-Dios y Pérez, 2007.

21. Porcel AM., Morales JM., Villaverde C, et al.). Nivel de Dependencia en Cuidados Hospitalarios: Validación de la Herramienta de la Gestión Iniciare®. En Instituto de Salud Carlos III (Ed). Libro de actas del XVI Encuentro Internacional de Investigación en Cuidados. Alicante: Instituto de Salud Carlos III. Unidad de Investigación en Cuidados de Salud (Investén-isciii). 2009. Recuperado de: http://www.isciii.es/ISCIII/es/contenidos/fd-el-instituto/fd-organizacion/fd-estructuradirectiva/fd-subdireccion-general-redes-centros-investigacion2/fd-centros-unidades2/fdinvesten-isciii2/docus/2009 XIII encuentro Investen Alicante.pdf

22. Lima-Serrano M., Lima-Rodríguez JS. y Sáez-Bueno A. Diseño y validación de dos escalas para medir la actitud hacia la alimentación y la actividad física durante la adolescencia, Revista Española de Salud Pública. 2012; 86(3): 253-68.

23. Lima-Rodríguez J., Lima-Serrano M., Jiménez-Picón N., e al. Consistencia interna y validez de un cuestionario para medir la autopercepción del estado de salud familiar, Revista Española de Salud Pública. 2012; 86(5): 509-21.

24. Robles L. y Vázquez-Garnica EK. El cuidado a los ancianos: valoraciones en torno al cuidado no familiar. Texto \& Contexto Enfermagem. 2008; 17(2): 225-31.

25. De Carvalho LC., Feuerwerker LCM. y Merhy EE. Disputas en torno a los planes de cuidado en la internación domiciliaria: una reflexión necesaria. Salud Colectiva. 2007; 3(3): 259-69. 